\title{
SUPORTE DE TECNOLOGIAS DA INDÚSTRIA 4.0 PARA ASSEGURAR A SUSTENTABILIDADE NO PROCESSO DE DESENVOLVIMENTO DE PRODUTOS
}

Natália Ferraz (nati.ferraz@gmail.com) - Universidade Federal de São Carlos/Departamento de Engenharia de Produção

José Carlos de Toledo (toledo@dep.ufscar.br) - Universidade Federal de São Carlos

\section{RESUMO}

A transição para indústria 4.0 é um desafio para muitas empresas. Muitos processos tendem a ser otimizados com as tecnologias emergentes da indústria 4.0, que poderão impactar na sustentabilidade de muitos processos industriais. O presente artigo mostra, por meio de uma revisão da literatura, como algumas tecnologias habilitadoras da indústria 4.0 podem assegurar a sustentabilidade no processo de desenvolvimento de produtos. Observou-se que as tecnologias abordadas neste estudo afetam de modo/intensidade diferente as etapas do PDP. As etapas de produção e manutenção são mais fortemente impactadas, do ponto de vista da sustentabilidade, pelas 3 tecnologias. As tecnologias com foco em tratamento e acesso a grandes volumes de dados (CC e BD) afetam mais intensamente nas etapas iniciais do PDP. Este, tratase de um tema que merece ser explorado em futuras pesquisas.

Palavras chave: Indústria 4.0; Tecnologias da indústria 4.0; Processo de desenvolvimento de produtos; sustentabilidade. 


\section{INTRODUÇÃO}

As empresas são constantemente desafiadas a lançar continuamente novos produtos antes da concorrência, exigindo Processos de Desenvolvimento de Produtos (PDP) mais ágeis e eficientes. Além disso, a preocupação com a sustentabilidade é cada vez mais presente nas decisões empresariais. No desenvolvimento do produto são tomadas as principais decisões que influenciam na sustentabilidade dos produtos e processos. A sustentabilidade requer mudanças nas organizações, as quais devem aprimorar o gerenciamento de suas operações de modo ecologicamente correto, economicamente viável e socialmente justo.

O emergente termo "indústria 4.0" tem se tornado um tópico de debates e discussões nos últimos anos. A Indústria 4.0 representa um sistema tecnológico complexo que introduz relevantes avanços relacionados às futuras fábricas inteligentes. Esse novo paradigma traz consigo tecnologias como os Sistemas Ciber-Físicos (CPS), Internet das Coisas (IoT), Internet de Serviços (IoS), Cloud Computing, Robótica, Big Data, Manufatura Aditiva, Realidade Aumentada, dentre outras fundamentais para o desenvolvimento e aprimoramento de produtos e processos mais inteligentes (PEREIRA e ROMERO, 2017).

Muitos estudos (SANTOS et al.,2017; TAO et al.,2018) investigam as influências de tais tecnologias no PDP. Mas, pouco se sabe sobre como elas ajudam as empresas a assegurarem a sustentabilidade no processo desenvolvimento de produtos. Diante disso o presente estudo visa investigar, por meio de revisão bibliográfica, como as tecnologias habilitadoras da indústria 4.0 auxiliam a assegurar a sustentabilidade no PDP.

O escopo deste artigo limita-se a investigação de três tecnologias habilitadoras da indústria 4.0: Cloud Computing (CC), Big Data (BD) e Manufatura aditiva (MA). Esse estudo foi realizado por meio de revisão bibliográfica a partir de artigos na base de dados Web of science adotandose palavras chaves associadas à indústria 4.0, processo de desenvolvimento de produto e sustentabilidade. A busca foi por artigos em língua inglesa e publicados entre 2014 a 2019. Espera-se, por meio dos resultados apresentados neste artigo, fornecer uma compreensão sobre as relações entre PDP, Sustentabilidade e Indústria 4.0, sendo este um relevante tópico para futuros estudos mais intensivos.

\section{REVISÃO TEÓRICA}




\subsection{Indústria 4.0}

Também conhecida como Quarta revolução industrial, o- conceito "Indústria 4.0” é um termo genérico para um novo paradigma que engloba um conjunto de futuros desenvolvimentos industriais em relação aos Sistemas Ciber-Físicos (CPS), Internet de Coisas (IoT), Internet de Serviços (IoS), Robótica, Big Data, Cloud Manufacturing e Realidade Aumentada. A adoção dessas tecnologias é fundamental para o desenvolvimento de processos mais inteligentes, que inclui dispositivos, máquinas, módulos de produção e produtos capazes de trocar informações de forma independente, acionar ações e controlam umas às outras, permitindo um ambiente de fabricação inteligente (WEYER et al, 2015).

A digitalização é uma tendência que influencia não só as empresas, mas a sociedade como um todo (OESTERREICH e TEUTEBERG, 2016). Um de seus aspectos mais evidente é o uso das tecnologias digitais para conectar pessoas, sistemas, empresas, produtos e serviços (LIAO_et al.2017; OESTERREICH e TEUTEBERG, 2016; PARIDA et al., 2019). O fortalecimento e a interação dessas diversas tecnologias (físicas e digitais) por meio da internet resultam em sistemas ciber-físicos e inteligentes, capazes de agregar valor para as operações e processos industriais (LIAO et al., 2017; MÜLLER et al., 2018).

Os produtos e processos estão sendo largamente influenciados por este novo paradigma industrial. As rápidas mudanças econômicas e a crescente dinâmica do mercado resultam numa demanda por desenvolvimento de produtos mais complexos e inteligentes. Ao mesmo tempo, os produtos se tornam mais modulares e configuráveis, possibilitando personalização em massa e impulsionando processos de desenvolvimento ágeis, criativos e colaborativos. Com as tecnologias digitais emergentes, os produtos passam a ser capazes de fornecer informações em tempo real sobre o estágio do ciclo de vida do produto, gerando benefícios para toda a cadeia de valor.

\subsection{O Processo de Desenvolvimento de Produtos (PDP)}

A medida que os cliente se tornam cada vez mais exigentes, surgindo a todo momento novas necessidades e desejos, cresce a demanda por novos produtos. Em paralelo a isso, as empresas necessitam ter alta qualidade, rapidez e baixo custo de desenvolvimento de novos produtos (PAULA e MELLO, 2013). Para que o desenvolvimento de produtos esteja conciliado com 
esses objetivos, se faz necessária a adoção de um processo sistemático de desenvolvimento de produtos, sendo este um fator crítico de sucesso no alcance dos objetivos estratégicos das empresas. O PDP consiste num conjunto de atividades que possibilitam determinar as especificações de projeto de um produto a partir da identificação das necessidades do mercado, considerando as oportunidades e restrições tecnológicas bem como os recursos de produção disponíveis de modo que o produto possa contribuir com a estratégia competitiva da organização (ROZENFELD et al, 2006).

Os modelos de referência para o gerenciamento do PDP são compostos por etapas sequencialmente definidas e padronizadas que podem ser aplicadas ao desenvolvimento de produtos diversos.

\begin{tabular}{|c|c|c|c|c|c|c|}
\hline \multicolumn{2}{|c|}{ Pré desenvolvimento } & \multicolumn{2}{|c|}{ Desenvolvimento } & \multicolumn{3}{|c|}{ Pós desenvolvimento } \\
\hline Requisitos & Definição & Design & Implementação & Produção & Manutenção & Retirada \\
\hline Anălise & Gera & Cria & $\begin{array}{l}\text { Realiza e } \\
\text { examina }\end{array}$ & Produz & Reparos & Recicla \\
\hline $\begin{array}{l}\text { exigência do } \\
\text { cliente }\end{array}$ & $\begin{array}{c}\text { lista de } \\
\text { necessidades } \\
\text { lista de recursos }\end{array}$ & $\begin{array}{c}\text { Modelo do } \\
\text { produto }\end{array}$ & Protótipo & Produtos & Produtos quebradc & \\
\hline
\end{tabular}

Figura 1 - Modelo de referência para Processo de Desenvolvimento de Produto. Fonte: Adaptado de Cooper (2001).

O Processo de Desenvolvimento de produtos, conforme o modelo de Cooper (2001) ilustrado na Figura 1, é dividido em sete fases. Na fase de requisitos ocorre a definição do conceito do produto que reflete o conjunto de necessidades dos clientes. A segunda fase, definição, envolve a tradução dos requisitos dos clientes em características do produto, estabelecidas de forma clara e objetiva. Em seguida inicia-se a fase de projeto (design) onde os modelos conceituais são detalhados e transformados posteriormente em um protótipo. O projeto passa a se concretizar na fase de implementação na qual o projeto do produto começa a ser executado e ocorre a sua visualização real. A fase de produção resulta no produto almejado com o custo estipulado. Posteriormente, na fase de manutenção realiza-se o monitoramento do produto, acompanhando todo o seu ciclo de vida. A fase de retirada refere-se à análise da retirada ou descontinuidade de produtos do mercado em razão da perda de interesse dos consumidores ou do alto custo das adaptações necessárias para o atendimento das novas exigências do mercado. A pesquisa voltada ao desenvolvimento de produtos tem atraído pesquisadores por várias décadas (KLEINSCHMIDT et al., 2007; PAGE, 1993; WIND e MAHAJAN, 1988) onde 
tópicos emergentes tais como inovação, engenharia, colaboração, processos de gestão e ferramentas facilitadoras da gestão do PDP tem sido discutidos. Dentre estes tópicos, a partir dos anos 2000 tem ocorrido um crescimento de estudos sobre a sustentabilidade no contexto do PDP (AHMAD et al, 2018; GMELIN e SEURING, 2014; SCHOGGL, BAUMGARTNER e HOFER, 2017; YOUNESI e ROGHANIAN, 2015), visto que as empresas têm sido estimuladas a desenvolver, fabricar e entregar produtos, sendo sustentáveis e ao mesmo tempo fornecendo um novo valor para o clientes que atendam as suas necessidades (GMELIN e SEURING, 2014)

\subsection{A sustentabilidade no processo de desenvolvimento de produto}

Nos últimos anos a sustentabilidade tem obtido grande destaque entre as publicações em variadas áreas da ciência (WANG et al., 2015). A sustentabilidade, conforme a definição mais aceita está relacionada com a trile bottom line (TBL) que se refere as três dimensões da sustentabilidade: economia, meio ambiente e sociedade. Assim, o conceito de sustentabilidade com base na TBL visa o equilíbrio por meio da lucratividade econômica, respeito ao meio ambiente e responsabilidade junto à sociedade (GMELIN e SEURING, 2014). No contexto do desenvolvimento de produto, a sustentabilidade relaciona-se ao esforço contínuo para obter a eficiência em projeto de produtos que resultem no desempenho almejado e que resultem em processos que busquem reduzir impactos ambientais e proporcionar simultaneamente benefícios sociais e econômicos aos interessados (AHMAD et al, 2018; BUCHERT et al., 2017). Um processo de desenvolvimento de produto sustentável concilia de forma eficaz as três dimensões da sustentabilidade. É importante destacar que a maioria dos estudos e metodologias disponíveis na literatura contemplam a sustentabilidade de forma parcial, sendo que a dimensão ambiental prevalece na maioria das abordagens metodológicas do processo de desenvolvimento de produto sustentável (AHMAD et al, 2018).

Uma variedade de ferramentas de eco-desing (Design for environment - DfE) foram criadas visando suportar o estágio de concepção do produto (NAVARRO et al., 2005; SHI et al., 2017). De acordo com Buchert et al (2017) metodologias tais como LCA - Lifecycle Assessment, QFD - Quality Function Deployment, LCC - Lifecycle Costing, TRIZ - Theory of Inventive Problem Solving, SLCA - Social Lifecycle Assessment são empregadas em métodos para desenvolvimento sustentável de produtos propostos por Crul e Diehl (2006), Finkbeiner et al.(2010), Dufrene et al. (2013) e Romli et al. (2015). Inserir a sustentabilidade no processo de 
desenvolvimento de produtos é um desafio, pois envolve vários critérios, requisitos, informações e variados conhecimentos que necessitam ser gerenciados e analisados com acurácia.

Além da preocupação com o desenvolvimento de novos produtos orientados para a sustentabilidade, as empresas lidam com ciclos de vida cada vez mais curtos e produtos cada vez mais inovadores, exigindo capacidades para criar novos produtos e lançá-los antes da concorrência. À medida que aumenta a preocupação em assegurar a sustentabilidade no PDP, a atividade de desenvolver produtos tem se tornado cada vez mais complexa (YIN e QIN, 2019) em razão dos fatores dinâmicos e das possibilidades de inserção de tecnologias aprimoradas para auxílio ao desenvolvimento de produtos.

\section{TECNOLOGIAS HABILITADORAS DA INDÚSTRIA 4.0 COMO SUPORTE À SUSTENTABILIDADE DO PDP}

\subsection{Manufatura aditiva/3D printing}

A manufatura aditiva (MA) surge como uma das tecnologias impulsionadoras de mudanças no cenário da manufatura que possibilita um maior custo-benefício e eficiência na produção em pequena escala (FORD e DESPEISSE, 2016). Também conhecida como tecnologia de impressão 3D, a MA pode ser definida como um processo de união de materiais para a criação de objetos por meio de modelos tridimensionais, cujo processo ocorre geralmente camada sobre camada, ao contrário dos métodos tradicionais (CERDAS et al, 2017).

O uso da MA está em constante crescimento, pois as empresas estão tentando reduzir ao máximo o consumo de matérias-primas e o intervalo de tempo entre projeto e fabricação, sem o comprometimento da qualidade dos produtos (CERDAS et al, 2017; MA et al., 2018).

A MA oferece vantagens em termos de mão de obra, materiais, lead-time entre projeto e fabricação e otimizam as atividades de prototipagem e projeto (COTTELEER e JOYCE, 2014), podendo ter mais rentabilidade do que a fabricação convencional, uma vez que a MA não envolve longos prazos de entrega, custos com estoques, componentes complexos e caros (WOHLERS, 2015).

Ford e Despeisse (2016) destacam três benefícios potenciais do efeito desta tecnologia na sustentabilidade: maior eficiência de recursos nas fases de produção e uso, como processos de 
fabricação e produtos que podem ser redesenhados para MA; extensão da vida útil do produto através de conserto, remanufatura e reforma; cadeias de valor reconfiguradas envolvendo cadeias de suprimentos mais curtas e simples, produção mais localizada, modelos de distribuição inovadores e novas formas de colaboração. Outras vantagens incluem a geração de um menor volume de resíduos durante a fabricação por se tratar de um processo aditivo; a capacidade de otimizar geometrias para a criação de componentes leves e adaptados ao produto reduzindo a demanda de material e consumo de energia; redução nos custos de transportes na cadeia de suprimentos e redução do nível de estoque devido a produção sob demanda (CHEN et al., 2015; MANI, 2014).

Gebler et al. (2014) apontam que são esperados ganhos econômicos devido a fatores tais como economias de energia, otimização de material e de manuseio, justificados pelas cadeias de suprimentos encurtadas.

No que diz respeito à dimensão social, poucos estudos investigaram os benefícios da MA para este contexto. Huang et al (2013) apontam a redução de trabalhadores em exposição à periculosidades. Outro aspecto diz respeito à democratização da produção que as tecnologias de MA fornecem, ocasionando mudanças nos padrões de consumo de modo que os consumidores se tornam cada vez mais ativos no PDP.

Há escassez de estudos que investigam os impactos sobre a sustentabilidade com o uso desta tecnologia na fase de projeto do produto. Ford e Despeisse (2016) realizaram um estudo exploratório sobre as vantagens e desafios da MA e suas implicações na sustentabilidade. Os autores avaliaram as implicâncias da MA sob o ponto de vista do ciclo de vida do produto, apesar de pouca ênfase ser dada a fase de projeto. Esse estudo trouxe algumas considerações sobre a sustentabilidade do uso da MA no estágio de projeto do produto:

- A flexibilidade em projeto possibilitada pela MA permite redesenhos no produto ou nos seus componentes. $\mathrm{O}$ redesenho pode atender aos requisitos funcionais ao mesmo tempo em que reduz a demanda de material necessário.

- Há uma redução do lead-time entre o projeto e a fabricação, visto que estes estarão diretamente interligados, o que também resultará em menores custos.

- Outra possibilidade de redução de custos diz respeito ao desenvolvimento de componentes modulares, capazes de explorar as potencialidades da MA. 
No que diz respeito ao estágio do projeto do produto, Ford e Despeisse (2016) apontaram algumas vantagens e desafios da sustentabilidade através MA (Quadro 1).

Quadro 1 - Vantagens e desafios de sustentabilidade da MA no estágio de projeto

\begin{tabular}{|c|c|}
\hline Vantagens & Desafios \\
\hline \multicolumn{2}{|c|}{ Projeto de produto } \\
\hline $\begin{array}{l}\text { - } \quad \text { Liberdade de projeto } \\
\text { - } \quad \text { Uso de conceitos de biomimética } \\
\text { - } \quad \text { Geometrias e desempenhos otimizados } \\
\text { para atender aos requisitos funcionais. } \\
\text { - } \quad \text { Desmatearialização do produto. } \\
\text { simplificados. } \\
\text { - } \quad \text { Problemas de custo, volume e qualidade } \\
\text { reduzidos através da montagem simplificada. } \\
\text { - } \quad \text { Redução de tempo entre design e fabricação } \\
\text { - } \quad \text { Melhoria na funcionalidade do produto } \\
\text { - } \quad \text { Maior durabilidade do produto } \\
\text { - } \quad \text { Atualização através do design modular } \\
\text { - } \quad \text { Processo de design democratizado }\end{array}$ & $\begin{array}{l}\text { - } \quad \text { Formação de designers e engenheiros sobre } \\
\text { os usos e benefícios potenciais da MA. } \\
\text { - Apoiar o desenvolvimento de habilidades de } \\
\text { designers e engenheiros. } \\
\text { - Integra considerações de sustentabilidade } \\
\text { usando os princípios do Design for Environment } \\
\text { (DfE) ou eco-design. } \\
\text { - Certificação de novos componentes } \\
\text { - Captura e replicação de aprendizado em } \\
\text { aplicações futuras. }\end{array}$ \\
\hline
\end{tabular}

Fonte: Ford e Despeisse (2016)

\subsection{Cloud Computing}

As tecnologias da computação em nuvem (CC) abrangem soluções de aplicações, plataformas e infraestruturua que são entregues como uma rede de serviços pública ou privada. A CC é capaz de aglomerar, armazenar e processar um grande volume de dados que serão analisados e estarão acessíveis em qualquer região de alcance, sem fronteiras físicas ou virtuais (SANTOS et al, 2017). Dentre suas principais vantagens, Shingh et al (2018) citam a redução de custos para as empresas que a utilizam, a rápida implantação dos recusos computacionais e a melhoria da visibilidade das informações.A personalização de produtos é apontada por Wu et al (2015) como uma das possibilidades advindas dos projetos baseado em núvem que permitem aos projetistas configurar, selecionar e utilizar recursos e serviços para desenvolver produtos conforme a necessidade do cliente. Os dados do projeto podem ser acessados por qualquer um e em qualquer lugar e podem ser instantemente compartilhados com parceiros. 


\begin{tabular}{|c|c|c|c|}
\hline & $\begin{array}{c}\text { Do ponto de vista } \\
\text { ambiental }\end{array}$ & $\begin{array}{l}\text { Do ponto de vista } \\
\text { econômico }\end{array}$ & $\begin{array}{l}\text { Do ponto de vista } \\
\text { social }\end{array}$ \\
\hline $\begin{array}{l}\text { Huang et al, } \\
2017\end{array}$ & $\begin{array}{l}\text { Permite armazenar e } \\
\text { compartilhar informação, } \\
\text { conhecimento e dados } \\
\text { detalhados do produto } \\
\text { para subsidirar o DfD } \\
\text { (Design para desmontagem) } \\
\text { para o melhor aproveitamen- } \\
\text { to dos resíduos. }\end{array}$ & $\begin{array}{l}\text { Torna as atividades de } \\
\text { desenvolvimento do produto } \\
\text { mais eficientes, possibilitando } \\
\text { a redução de custos neste } \\
\text { processo. }\end{array}$ & $\begin{array}{l}\text { Projeto colaborativo - Os proje- } \\
\text { tistas podem acessar o serviço a } \\
\text { qualquer hora ou lugar, compar- } \\
\text { tilhar dados e colaborar com } \\
\text { parceiros prontamente }\end{array}$ \\
\hline $\begin{array}{c}\text { Alptekin e } \\
\text { Alptekin, } 2018\end{array}$ & $\begin{array}{l}\text { Menor uso de recusos (por } \\
\text { exemplo energia elétrica). } \\
\text { Redução no preço de serviços de } \\
\text { projeto fornecidos por meio da } \\
\text { computação em núvem. }\end{array}$ & $\begin{array}{l}\text { Maior economia de recursos } \\
\text { computacionais. } \\
\text { Inclusão de conceitos como } \\
\text { extensibilidade e remanufatu- } \\
\text { rabilidade do produto projeta- } \\
\text { do. } \\
\text { Auxilia no projeto do produto } \\
\text { com melhor relação } \\
\text { custo-benefício. }\end{array}$ & $\begin{array}{l}\text { Foco na satisfação dos consumi- } \\
\text { dores e de todas as partes inte- } \\
\text { ressadas } \\
\text { Projeto orientado ao cliente. } \\
\text { Capaz de atender a segmentos } \\
\text { diversos de clientes. }\end{array}$ \\
\hline $\begin{array}{l}\text { Singh et al, } \\
2018\end{array}$ & $\begin{array}{l}\text { Oferece suporte na seleção e in- } \\
\text { clusão de fornecedores ecológica- } \\
\text { mente corretos para a tomada de } \\
\text { decisão sobre materiais (fase de- } \\
\text { finição e projeto) por meio contro- } \\
\text { le e análise de dados de indicado- } \\
\text { res ambientais (exemplo: emis- } \\
\text { são CO2) }\end{array}$ & $\begin{array}{l}\text { Redução do custo para a } \\
\text { empresa } \\
\text { Economia na utilização recursos } \\
\text { fornecidos por provedores em } \\
\text { núvem. } \\
\text { Possibilita selecionador fornece- } \\
\text { dores de insumos de alta qualida- } \\
\text { de e menor custo }\end{array}$ & $\begin{array}{l}\text { Compartilhamento de informa- } \\
\text { ções e recursos com as partes } \\
\text { interessadas. }\end{array}$ \\
\hline
\end{tabular}

Fonte: os autores (2019).

A importancia da sustentabilidade com o uso das tecnologias em núvem fica cada vez mais evidente (ALPTEKIN e ALPTEKIN, 2018; HUANG et al 2017), sendo este um tópico emergente de pesquisa. Baseado em arquitetura de $\mathrm{CC}$, os projetistas armazenam e trocam informações e conhecimento de projeto para direcionar o desenvolvimento sustentável de produtos (HUANG et al, 2017). Algumas aplicações de CC no desenvolvimento de produtos que ajudam assegurar a sustentabilidade são destacadas no Quadro 2.

\subsection{Big Data e Analytic}

A capacidade de coletar, organizar e analisar enormes quantidades de dados de fontes diversas é um dos grandes destaques dentre as tecnologias da Indústria 4.0. A aplicação de Big Data e Data Analytic otimiza a qualidade da produção, economiza energia e melhora o desempenho dos equipamentos. O termo Big Data (BD) refere-se a uma abordagem voltada a capacidade de agregar, coletar e extrair um grande volume de dados para auxiliar na tomada de decisões sobre emergentes sistemas complexos (LADEAU et al, 2017). 
Todos os estágios do ciclo de vida de um produto geram um grande volume de dados que necessitam ser gerenciados (TAO et al, 2017). Considera-se dois estágios fundamentais no processo de desenvolvimento de um produto: a Geração do conceito e o Projeto detalhado do produto em virtude da limitação do foco deste estudo. O Quadro 3 resume os benefícios das aplicações do BD para os estágios de Geração do conceito e Projeto detalhado do produto.

\section{Quadro 3 - Benefícios do BD na geração do conceito e Projeto do produto}

\begin{tabular}{|l|l|}
\hline \multicolumn{1}{|c|}{ Geração do conceito } & \multicolumn{1}{|c|}{ Projeto do produto } \\
\hline $\begin{array}{l}\text { Com base nas demandas dos clientes, informações } \\
\text { de mercado, planejamento de investimentos e } \\
\text { outros dados, define-se o conceito de novos produtos } \\
\text { ou melhorias no design do produto, bem como a } \\
\text { estética e as principais funções do produto. Nesse } \\
\text { estágio, vários dados precisam ser processados, } \\
\text { como várias formas de demandas de clientes, } \\
\text { incluindo comentários, reclamações e vídeos na } \\
\text { Internet, informações de mercado, volume de vendas } \\
\text { de produtos, satisfação do cliente, planejamento de } \\
\text { investimentos e assim por diante. }\end{array}$ & $\begin{array}{l}\text { A equipe de desenvolvimento de produto conclui o } \\
\text { trabalho de projeto de produto de forma colaborativa } \\
\text { por meio da troca e compartilhamento de dados e } \\
\text { ideias de projeto. Os dados envolvidos no projeto do } \\
\text { produto incluem a descrição da função e aparência } \\
\text { do produto, configurações do produto, parâmetros de } \\
\text { projeto e dados de teste, etc. E até mesmo dados } \\
\text { históricos de falhas de produtos similares podem } \\
\text { ajudar a melhorar o projeto do produto. }\end{array}$ \\
\hline
\end{tabular}

Fonte: Adaptado de Tao et al (2017)

A sustentabilidade nos estágios de geração de conceito e projeto pode ser assegurada com o suporte de BD, como explanado por Singh et al (2018) por meio de estudo de caso realizado em uma cadeia de suprimentos bovina para a seleção de fornecedores ecologicamente corretos. O BD foi utilizado para capturar, cruzar e analisar um grande volume de dados para selecionar aqueles fornecedores com base em indicadores ambientais e econômicos, a partir do propósito de desenvolver produtos de qualidade, a custos acessíveis e ambientalmente corretos.

A gestão de dados por meio de BD tornam as atividades mais rápidas e mais confiáveis na geração do conceito e na fase de projeto do produto, o que possibilita a geração de economias no PDP. Além disso, favorece a escolha de matérias-primas mais sustentáveis que comporão os produtos. Do ponto de vista social, também dá suporte ao projeto colaborativo e possibilita o compartilhamento instantâneo de um grande volume de dados com as partes interessadas. Essa tecnologia oferece melhores resultados, conforme Singh et al (2018), se somada a CC.

Conforme estudos realizados na literatura sobre esta temática emergente, observou-se que as tecnologias em questão possuem diferentes contribuições em cada fase do PDP. 
Quadro 4 -Relação entre as tecnologias habilitadoras da I4.0 com a sustentabilidade nas fases do PDP

\begin{tabular}{|c|c|c|c|c|c|c|c|}
\hline & Requisitos & Definição & $\begin{array}{l}\text { Projeto/ } \\
\text { Design }\end{array}$ & Implementação & Produção & Manutenção & Retirada \\
\hline $\begin{array}{l}\text { Manufatura } \\
\text { aditiva }\end{array}$ & & & & & & & \\
\hline $\begin{array}{l}\text { Cloud } \\
\text { Computing }\end{array}$ & & & & & & & \\
\hline Big Data & & & ( & & & & \\
\hline
\end{tabular}

Fonte: os autores (2019)

Conforme o Quadro 4 observa-se que as 3 tecnologias afetam de modo/intensidade diferente as etapas do PDP. As etapas de produção e manutenção são mais fortemente impactadas, do ponto de vista da sustentabilidade, pelas 3 tecnologias. As tecnologias com foco em tratamento e acesso a grandes volumes de dados (CC e BD) afetam mais intensamente nas etapas iniciais do PDP.

O Quando 5 sumariza como as tecnologias da indústria 4.0 podem assegurar a sustentabilidade no PDP. 


\begin{tabular}{|c|c|c|c|}
\hline Dimensão & Cloud Computing & Big Data & Manufatura aditiva \\
\hline AMBIENTAL & \begin{tabular}{|l|} 
Permite armazenar e compartilhar informação, \\
conhecimento e dados detalhados do produto \\
para subsidiar a prática de projeto para facilitar \\
a desmontagem no final da vida do produto \\
(reciclagem). \\
Auxilia a prática de projeto modular, que reduz \\
o uso de insumos e o aumento do ciclo de vida \\
do produto. \\
Facilita a inclusão de conceitos como extensi- \\
bilidade e remanufaturabilidade do produto pro- \\
jetado. \\
Permite melhor aproveitamento de componen- \\
tes, estendendo o ciclo de vida do produto. \\
Permite um menor uso de recursos, como energia \\
elétrica.
\end{tabular} & $\begin{array}{l}\text { Apoia ao gerenciamento do ciclo de vida do produ- } \\
\text { to. } \\
\text { Permite capturar, cruzar e analisar um grande volu- } \\
\text { me de dados para selecionar fornecedores e mate- } \\
\text { riais a serem inseridos no projeto do produto com } \\
\text { base em indicadores ambientais. }\end{array}$ & $\begin{array}{l}\text { Maior eficiência de recursos nas fases projeto e } \\
\text { produção, como processos de fabricação e produ- } \\
\text { tos podem ser redesenhados para AM. } \\
\text { Maior vida útil do produto através de conserto, re- } \\
\text { manufatura e reforma. } \\
\text { Geração de menos residuos, por se tratar de um } \\
\text { processo aditivo. } \\
\text { Menor demanda de recursos, como energia elétrica. } \\
\text { Simplifica a manufatura e montagem de produtos } \\
\text { Maior durabilidade do produto. } \\
\text { Permite a atualização do produto através do uso do } \\
\text { conceito de projeto modular }\end{array}$ \\
\hline ECONÔMICA & $\begin{array}{l}\text { Maior eficiência do PDP possibilitando a redução } \\
\text { de custos. } \\
\text { Maior economia de recursos computacionais. } \\
\text { Melhora a eficiência de um departamento de pro- } \\
\text { jeto/desenvolvimento de produto, tornando esse } \\
\text { processo menos oneroso. }\end{array}$ & $\begin{array}{l}\text { Gerencia e analisa dados sobre indicadores eco- } \\
\text { nômicos a serem considerados no projeto do pro- } \\
\text { duto. } \\
\text { Auxilia na tomada de decisões sobre fornecedores } \\
\text { e componentes com melhor relação custo/beneficio. }\end{array}$ & $\begin{array}{l}\text { Problemas de custo, volume e qualidade são redu- } \\
\text { zidos através da montagem simplificada. } \\
\text { Redução do intervalo de tempo entre o projeto e a } \\
\text { fabricação, tornando o processo mais rentável. } \\
\text { Beneficios econômicos provenientes da melhoria } \\
\text { nos processos. } \\
\text { Economia de material e possibilidade de reutilizar } \\
\text { recursos subjacentes ao processo de fabricação. } \\
\text { Produção direta de modelos CAD 3D elimina a ne- } \\
\text { cessidade de ferramentas e moldes, então não há } \\
\text { custos de troca. } \\
\text { Melhora a eficiência energética do processo. } \\
\text { Redução de custos do ciclo de vida do produto de- } \\
\text { corrente da otimização de material e de manuseio, } \\
\text { sendo este último em virtude das cadeias de supri- } \\
\text { mentos encurtadas. }\end{array}$ \\
\hline SOCIAL & $\begin{array}{l}\text { Possibilita o projeto colaborativo de modo que os } \\
\text { projetistas possam acessar o projeto do produto, } \\
\text { compartilhar dados do projeto e colaborar com par- } \\
\text { ceiros prontamente. } \\
\text { Permite projeto orientado ao cliente, com maior } \\
\text { personalização de produtos e serviços. } \\
\text { Foco na satisfação do consumidor e das partes in- } \\
\text { teressadas. } \\
\text { Melhoria da visibilidade das informações pela equi- } \\
\text { pe de projeto e entre as partes interessadas. }\end{array}$ & $\begin{array}{l}\text { Gerenciamento e processamento de dados da de- } \\
\text { manda dos clientes e do mercado para subsidiar a } \\
\text { identificação de requisitos e a definição do conceito } \\
\text { do produto. } \\
\text { Possibilita o projeto colaborativo entre a equipe de } \\
\text { desenvolvimento de produto (troca e compartilha- } \\
\text { mento de dados e ideias de design). }\end{array}$ & $\begin{array}{l}\text { Geometrias e desempenhos otimizados para aten- } \\
\text { der aos requisitos especificos do cliente (persona- } \\
\text { lização). } \\
\text { Processo de projeto democratizado, com os con- } \\
\text { sumidores desempenhando papel mais ativo no } \\
\text { PDP. } \\
\text { Flexibilidade de produzir em qualquer lugar e por } \\
\text { qualquer pessoa. } \\
\text { Consumidores individuais podem projetar e produ- } \\
\text { zir produtos em sua conveniência } \\
\text { Redução de trabalhadores em exposição a pericu- } \\
\text { losidades. } \\
\text { Facilidade de aquisição de algumas tecnologias de } \\
\text { MA, permitindo produção descentralizada e locali- } \\
\text { zada. }\end{array}$ \\
\hline
\end{tabular}

Fonte: os autores (2019)

\section{CONCLUSÃO}

A indústria 4.0 põe em destaque um conjunto de tecnologias habilitadoras que vem modificando a configuração do ambiente de negócio das organizações. A pesquisa na literatura aponta que tecnologias habilitadoras da indústria 4.0, tais como BD, CC e MA, são capazes de trazer benefícios diretos e indiretos em termos de sustentabilidade no PDP. A MA por ser categorizada como uma megatendência digital apresenta contribuições mais tangíveis e mais diretas no que diz respeito ao potencial de suporte à sustentabilidade no PDP. Dentre os principais benefícios destaca-se a economia de materiais, a eficiência energética, o compartilhamento instantâneo de dados do projeto e motiva o projeto colaborativo. A $C C$ permite benefícios como o compartilhamento em tempo real do projeto ou seus respectivos dados e facilita a armazenagem de dados sobre indicadores ambientais para a seleção de materiais e fornecedores mais sustentáveis. O Big Data facilita e otimiza o gerenciamento de dados durante todo o ciclo de vida do produto e torna as atividades de geração de conceito e projeto do produto mais rápidas 
e confiáveis, principalmente para produtos que envolvem decisões com variados requisitos, que geram um grande volume de dados.

Como futuras pesquisas sugere-se explorar as demais tecnologias da indústria 4.0 para uma maior compreensão sobre como exercerão influência na sustentabilidade do PDP. Para uma investigação mais extensiva, outras bases de dados além da base Web of Science deve ser exploradas.

\section{REFERÊNCIAS}

AHMAD, Shamraiz et al. Sustainable product design and development: A review of tools, applications and research prospects. Resources, Conservation and Recycling, v. 132, p. 49-61, 2018.

ALPTEKIN, S. Emre; ALPTEKIN, Gülfem Isiklar. A Fuzzy Quality Function Deployment Approach for Differentiating Cloud Products. International Journal of Computational Intelligence Systems, v. 11, n. 1, p. 1041-1055, 2018.

BUCHERT, Tom et al. Target-driven selection and scheduling of methods for sustainable product development. Journal of cleaner production, v. 161, p. 403-421, 2017.

CERDAS, Felipe et al. Life cycle assessment of 3D printed products in a distributed manufacturing system. Journal of Industrial Ecology, v. 21, n. S1, p. S80-S93, 2017.

CHEN, Danfang et al. Direct digital manufacturing: definition, evolution, and sustainability implications. Journal of Cleaner Production, v. 107, p. 615-625, 2015

COOPER, Robert Gravlin; EDGETT, Scott J.; KLEINSCHMIDT, Elko J. Portfolio management for new products. 2001.

COTTELEER, Mark; JOYCE, Jim. 3D opportunity: Additive manufacturing paths to performance, innovation, and growth. Deloitte Review, v. 14, p. 5-19, 2014.

DE PAULA, Jefferson Olegário; MELLO, Carlos Henrique Pereira. Seleção de um modelo de referência de PDP para uma empresa de autopeças através de um método de auxílio à decisão por múltiplos critérios. Production, v. 23, n. 1, p. 144-156, 2013.

FORD, Simon; DESPEISSE, Mélanie. Additive manufacturing and sustainability: an exploratory study of the advantages and challenges. Journal of Cleaner Production, v. 137, p. 1573-1587, 2016.

GEBLER, Malte; UITERKAMP, Anton JM Schoot; VISSER, Cindy. A global sustainability perspective on 3D printing technologies. Energy Policy, v. 74, p. 158-167, 2014.

GMELIN, Harald; SEURING, Stefan. Determinants of a sustainable new product development. Journal of Cleaner production, v. 69, p. 1-9, 2014.

HUANG, Samuel H. et al. Additive manufacturing and its societal impact: a literature review. The International Journal of Advanced Manufacturing Technology, v. 67, n. 5-8, p. 1191-1203, 2013.

LIAO, Yongxin et al. Past, present and future of Industry 4.0-a systematic literature review and research agenda proposal. International journal of production research, v. 55, n. 12, p. 3609-3629, 2017.

MA, Junfeng et al. An exploratory investigation of additively manufactured product life cycle sustainability assessment. Journal of Cleaner Production, v. 192, p. 55-70, 2018. 
MANI, Mahesh; LYONS, Kevin W.; GUPTA, S. K. Sustainability characterization for additive manufacturing. Journal of research of the National Institute of Standards and Technology, v. 119, p. 419, 2014.

NAVARRO, T. G. et al. Ecodesign function and form-classification of ecodesign tools according to their functional aspects. In: DS 35: Proceedings ICED 05, the 15th International Conference on Engineering Design, Melbourne, Australia, 15.-18.08. 2005. 2005. p. 605-606 (exec. Summ.), full paper no. DS35_346. 34.

OESTERREICH, Thuy Duong; TEUTEBERG, Frank. Understanding the implications of digitisation and automation in the context of Industry 4.0: A triangulation approach and elements of a research agenda for the construction industry. Computers in Industry, v. 83, p. 121-139, 2016.

ROZENFELD, Henrique et al. Gestão de desenvolvimento de produtos: uma referência para a melhoria do processo. 2006.

SCHÖGGL, Josef-Peter; BAUMGARTNER, Rupert J.; HOFER, Dietmar. Improving sustainability performance in early phases of product design: A checklist for sustainable product development tested in the automotive industry. Journal of Cleaner Production, v. 140, p. 1602-1617, 2017.

Shi, J., Li, Q., Li, H., Li, S., Zhang, J., Shi, Y. Eco-design for recycled products: rejuvenating mullite from coal fly ash. Resour. Conserv. Recycl. 124, 67-73. 2017.

SINGH, Akshit et al. Big data cloud computing framework for low carbon supplier selection in the beef supply chain. Journal of cleaner production, v. 202, p. 139-149, 2018.

WANG, Zhen et al. Composite sustainable manufacturing practice and performance framework: Chinese auto-parts suppliers' perspective. International Journal of Production Economics, v. 170, p. 219-233, 2015.

WEYER, Stephan et al. Towards Industry 4.0-Standardization as the crucial challenge for highly modular, multi-vendor production systems. Ifac-Papersonline, v. 48, n. 3, p. 579-584, 2015.

WU, Yung-Hung; HO, Chao Chung. Integration of green quality function deployment and fuzzy theory: a case study on green mobile phone design. Journal of Cleaner production, v. 108, p. 271-280, 2015.

YIN, Yuanyuan; QIN, Sheng-feng. A smart performance measurement approach for collaborative design in Industry 4.0. Advances in Mechanical Engineering, v. 11, n. 1, p. $1687814018822570,2019$.

YOUNESI, Mojdeh; ROGHANIAN, Emad. A framework for sustainable product design: a hybrid fuzzy approach based on quality function deployment for environment. Journal of Cleaner Production, v. 108, p. 385-394, 2015. 